\title{
Explorando manifestações de simbolismo sonoro em três línguas Tupí: Suruí Paitér, Mbyá Tambeopé e Nhandeva Txiripá
}

\author{
Ana Suelly Arruda Câmara Cabral ${ }^{1}$ \\ Joaton Suruí ${ }^{2}$ \\ Mauro Luiz Carvalho ${ }^{3}$ \\ Uraan Anderson Suruí ${ }^{4}$
}

\begin{abstract}
This article deals with sound symbolism in three Tupian languages, Suruí Paitér, Nhandéwa Txiripá and Mbyá Tambeopé. One of the main focus is the process of ablaut involved in the expression of physical and psychological attenuation of the semantic of Suruí Paitér words. It is argued that attenuation in these languages is controlled by the speaker's will and is pragmatically motivated. The data supporting the analysis corroborate the view that high vowels and palatalization are common in sound symbolism expressions of diminutives and attenuatives across languages (Sapir 1911, 1929; Ultan 1978).
\end{abstract}

Keywords: Sound symbolism. Attenuative. Ablaut. Suruí Paitér. Nhandéwa Txiripá. Mbyá Tambeopé. Tupian Stock.

\section{Resumo}

Este artigo trata de simbolismo sonoro em três línguas Tupí: Suruí Paitér, Nhandéwa Txiripá e Mbyá Tambeopé. Um dos focos principais é o processo de ablaut envolvido na expressão de atenuação física e psicológica da semântica das palavras em Suruí Paitér. É argumentado que atenuação nessas línguas é controlada pela vontade do falante e pragmaticamente motivada. Os dados que fundamentam o estudo corroboram a visão de que vogais altas e palatalização são expressões comuns de simbolismo associados a diminutivos e atenuativos através das línguas (Sapir 1911, 1929; Ultan 1978).

Palavras-chave: Simbolismo sonoro. Atenuativo. Ablaut. Suruí Paitér. Nhandéwa Txiripé. Mbyé Tambeopé. Tronco Tupí.

\section{Introdução}

Neste artigo abordamos expressões de atenuação presentes em três línguas do tronco Tupí, o Suruí Paitér (fam. Mondé), o Nhandéwa Txiripá e o Mbyá

$1 \mathrm{LALI} / \mathrm{UnB}$, bolsista de produtividade científica do CNPq.

2 Aluno do Curso de Licenciatura em Educação Básica Intercultural, UNIR/Ji-Paraná.

3 Mestrando em Linguística, LALI/UnB, PPGL/IL/UnB.

4 Aluno do Curso de Licenciatura em Educação Básica Intercultural, UNIR/Ji-Paraná. 
Tambeopé (sub-ramo I da fam. ling. Tupí-Guaraní). ${ }^{5}$ Embora essa modalidade aspectual de atenuativo seja expressa nas três línguas por meio de estratégias distintas - em Suruí se manifesta principalmente por meio de um processo envolvendo ablaut, associado ou não a inserções ou modificações de consoantes, mas também pela combinação de temas com sufixos de semântica atenuativa, enquanto que em Nhandéwa Txiripá e em Mbyá Tambeopé se manifesta por meio de um processo derivacional de sufixação de um único morfema, com uma única realização fonético-fonológica -, as aplicações dessa categoria nas três línguas compartilham propriedades distribucionais similares. Por um lado, afetam a semântica de palavras distintas - nomes, verbos, advérbios, entre outros -, e por outro lado, podem atenuar dimensões tanto físicas quanto psicológicas dos argumentos, assim como atenuar a duração dos intervalos temporais em que os estados e eventos ocorrem, ou, ainda, minimizar o efeito de uma ação verbal sobre um objeto.

Os processos de atenuação ora abordados são de interesse para os estudos linguísticos Tupí, por afetarem a semântica de diferentes tipos de palavras e por se manifestarem em Suruí também por meio de ablaut, um processo atípico no agrupamento genético Tupí. Embora processos de simbolismo sonoro envolvendo consoantes e/ou vogais na expressão de aumentativos e diminutivos sejam encontrados em outras línguas do mundo (cf. Hinton, Nichols and Ohalla 1994:4; Silverstein 1994), o vocalismo do Suruí envolvendo ablaut, até onde é sabido, também não é típico de outros agrupamentos genéticos do Brasil, como o tronco Macro-Jê, as famílias Pano, Aruák e Karíb. Por ser um processo pouco comum nessa parte do mundo, passa a ser de interesse, tanto em uma perspectiva sincrônica - tipológica e areal -, quanto em uma perspectiva diacrônica, visto que envolve também questões relativas à gênese de processos dessa natureza através das línguas.

O presente estudo beneficiou-se particularmente dos trabalhos de Jakobsen (1994) sobre o vocalismo dos vocativos em Nootka, assim como dos estudos de Sapir $(1911 ; 1929)$ e de Jespersen (1933) sobre simbolismo sonoro, e da abordagem tipológica proposta por Hinton, Nichols e Ohala (1994) sobre esse tema.

Como observado por Hinton, Nichols e Ohala (op. cit.), simbolismo sonoro é o processo através do qual certas vogais, consoantes e suprassegmentos são escolhidos para consistentemente representarem propriedades visuais, tácteis, ou propriedades de objetos, como dimensão e forma. Por exemplo, segmentos tais quais consoantes palatais são frequentemente usados como formas diminutivas.

5 A língua Suruí é falada pelos Paitér ey, 'pessoas de verdade', que vivem em Rondônia, na Terra Indígena Sete de Setembro. Os Paitér ey são também conhecidos como Suruí, nome que lhes foi atribuído pelos não índios durante o contato, na década de 1960. Já a língua Mbyá Tambeopé é falada no Espírito Santo, Paraná, São Paulo, Rio de Janeiro, Santa Catarina, Rio Grande do Sul, Pará e Maranhão. E o Nhandéwa? 
A discussão que aqui apresentamos ${ }^{6}$ é uma exploração inicial sobre o modo e a intensidade da manifestação das relações entre som e significado em línguas Tupí. Embora inicial, mostra a importância do simbolismo sonoro nessas línguas e contribui em vários aspectos para reforçar ideias como a de Sapir (1994) sobre a tendência das línguas de escolherem certos segmentos e processos fonológicos para representar dimensões.

\section{Expressões atenuativas em Suruí Paitér}

A língua Paitér faz largo uso de processo de ablaut para atenuar referentes de nomes, assim como para atenuar estados, ações e processos verbais. Para Van der Meer (1985), trata-se, nos nomes, de expressão do diminutivo e nos verbos marca a concordância de diminutivo com respeito ao sujeito de intransitivos e objeto de transitivos. Consoante Van der Meer, quando o sujeito é pequeno, o verbo intransitivo pode ir para o diminutivo, enquanto que verbos transitivos só são marcados para diminutivo quando o objeto é pequeno. Ainda segundo Van der Meer, o diminutivo é marcado pela elevação de vogais e consoantes dentais na última sílaba da raiz ou no sufixo, ou, ainda, na penúltima sílaba. O diminutivo é marcado, segundo Van der Meer, em verbos, nomes, adjetivos, intensificadores, quantificadores, numerais, demonstrativos, em palavras que expressam tempo, aspecto e, possivelmente, outras classes, mas pode também ser marcado por meio de um sufixo especial que significa pequeno.

$\mathrm{Na}$ análise que ora apresentamos das expressões atenuativas nessa língua damos ênfase ao processo de ablaut, exemplificando-o maximamente e demonstrando que, quando marca verbos transitivos, não significa necessariamente que o referente do objeto é de dimensão pequena, e quando marca verbos intransitivos, também não corresponde necessariamente a sujeito com referente de forma pequena. Na nossa análise, trata-se de expressão de atenuação que pode estar relacionada a dimensões físicas ou psicológicas, afetivas. Seu uso é pragmaticamente motivado e decorre de uma escolha do falante, quando este quer ressaltar a atenuação. Em verbos transitivos significa na maioria das vezes que a ação verbal afetou ou vai afetar o objeto minimamente, embora, em certos casos relaciona-se à dimensão pequena do referente.

6 Os dados Suruí que serviram de base para o presente estudo foram extraídos de textos coletados ao longo de oito oficinas, realizadas entre 2007 e 2011, sob a coordenação de lideranças G̃apg̃ir. Estas oficinas foram planejadas para a normatização da escrita Paitér e registro de conhecimentos tradicionais, tendo em vista a produção de materiais em língua Suruí para as escolas das aldeias. Os dados da língua Mbyá Tambeopé foram coletados por Mauro Luiz Carvalho junto a Antônio Carvalho, liderança Guaraní do Espírito Santo. Os dados do Nhandéwa Txiripá foram coletados por esse mesmo pesquisador junto a seus parentes. 
Descrevemos em seguida a distribuição da expressão de ablaut em diferentes classes de palavras em Suruí, ressaltando mudanças fonológicas e prosódicas concomitantes.

\section{Atenuação por meio de ablaut}

Os dados analisados mostram que o processo de ablaut consiste na mudança principalmente em $u(\mathfrak{i})$ das vogais $a, e, o$ da sílaba inicial, medial ou final de um tema. Note-se, contudo, que o caso mais frequente é o de ablaut na sílaba final de um tema. Há casos em que o ablaut se dá concomitantemente com a inserção de uma consoante final. Pode também se dar concomitantemente com a palatalização da consoante imediatamente contígua à esquerda ou com a mudança de um $j$ final em uma oclusiva. Há ainda outros tipos de mudanças fonológicas e prosódicas que se associam ao ablaut, em tela, como mostraremos nas seções seguintes.

\section{Expressão do "atenuativo" por meio da mudança em $\boldsymbol{u}(\mathbf{i})$ das vogais $a$, $e$, o finais de um tema}

Esta é a estratégia mais comum para atenuar dimensões físicas e psicológicas.

Em nomes:

$\begin{array}{ll}\text { ikahp } & \text { 'dente' } \\ \text { ikuhp } & \text { 'dentinho' } \\ \text { kaboahp } & \text { 'chocalho' } \\ \text { kabouhp } & \text { 'chocalhinho' } \\ \text { akapeahp } & \text { 'mulher em reclusão' } \\ \text { akapeuhp } & \text { 'mulher mais jovem em reclusão' } \\ \text { lobeah } & \text { 'tipo de panela Paitér' } \\ \text { lobeup } & \text { 'tipo de panela com características da panela lobeah, mas menor do } \\ & \text { que esta' } \\ \text { wãap } & \text { 'buraco' } \\ \text { wãup } & \text { 'buraquinho' } \\ \text { ihp pekap } & \text { 'tábua' } \\ \text { ihp pekup } & \text { 'tábuazinha } \\ \text { inak } & \text { 'fezes' } \\ \text { inuk } & \text { 'fezezinhas' } \\ \text { ikãy } & \text { 'velho' } \\ \text { ikũy } & \text { 'velhinho' }\end{array}$




$\begin{array}{ll}\text { mokãy } & \text { 'fogo' } \\ \text { mokũy } & \text { 'foguinho' } \\ \text { txãy } & \text { 'moça' } \\ \text { txũy } & \text { 'mocinha' } \\ \text { sogãy } & \text { 'coisa velha' } \\ \text { sogũy } & \text { 'coisa velha pequenina' }\end{array}$

O exemplo seguinte mostra, além do ablaut, a inserção de uma consoante $t$ final.

beya 'avó'

beyut 'avozinha'

Há casos, como o exemplo seguinte, em que o ablaut afeta uma vogal longa reduzindo-lhe uma mora, mas com inserção concomitante de uma consoante $t$ final.

ixaah 'perda'

ixaut 'perdinha'

No exemplo para 'pé' seguinte, a vogal original muda para $i$ e não para $u$, como esperado, provavelmente em decorrência de assimilação com a qualidade anterior da vogal da sílaba precedente.

mipeh 'pé'

mipit 'pezinho'

No exemplo seguinte 'coisa grande', o ablaut é acompanhado de uma mudança na consoante final, afetando-lhe o modo e o ponto de articulação:

soboy 'coisa grande'

sobuk 'coisa pequenininha'

Note-se que em nomes que pertencem a classes semânticas de relações de parentesco e de certos nomes de animais, o atenuativo se manifesta por meio do sufixo atenuativo $-x i n \sim-i n$ :

$\begin{array}{ll}\text { arimẽ } & \text { 'macaco' } \\ \text { arimẽ xin } & \text { 'macaquinho' } \\ \text { wao } & \text { 'jacaré' } \\ \text { wao xin } & \text { 'jacarezinho' } \\ \text { amõ } & \text { 'vovô' } \\ \text { amõ xin } & \text { 'vovozinho' }\end{array}$


$\begin{array}{ll}\operatorname{moy} & \text { 'filho' } \\ \text { moy xin } & \text { 'filhinho' }\end{array}$

Há uma subclasse semântica cuja atenuação se faz por meio do morfema atenuativo $k u p / p u k$, que expressa a ideia de filhote, e que se combina com vários nomes:

$\begin{array}{ll}\text { lap } & \text { 'casa' } \\ \text { lap gup } & \text { 'casinha' } \\ \text { îh } & \text { 'rede' } \\ \text { îh kup } & \text { 'redinha' }\end{array}$

Nomes de animais podem combinar-se também com morfema que significa 'filhote' para expressar dimensão física pequena, mas também afetividade.

arimẽ 'macaco'

arimẽ puk 'filhote de macaco'

morip 'peixe'

morip muk 'peixinho'

\section{Atenuação de adjetivos}

Referentes de nomes de qualidades e de sensações são atenuados por meio de palatalização da consoante em posição de onset da sílaba final:

xadop 'quente'

xajop 'quentinho'

ou pelo ablaut da vogal final, com a inserção de uma consoante:

kerea 'claro'

kereut 'clarinho'

\section{Verbos intransitivos}

O exemplo seguinte mostra que em verbos intransitivos o ablaut pode afetar a primeira vogal do tema, como ocorre também em certos nomes:

txoba 'cuspir'

txobu 'cuspir pouco'

A atenuação pode ser expressa também conjuntamente por ablaut e pelo acréscimo do sufixo $\tilde{\imath}$ :

aahta 'deitado'

aut $\tilde{1} \quad$ 'deitadinho' 
ou pela palatalização de n e pela inserção de uma consoante final:

$\begin{array}{ll}\text { ãnota } & \text { 'em pé' } \\ \text { ãnyot ĩ } & \text { 'em pezinho' }\end{array}$

Nos verbos transitivos, a atenuação por meio de ablaut pode afetar a última vogal do tema:

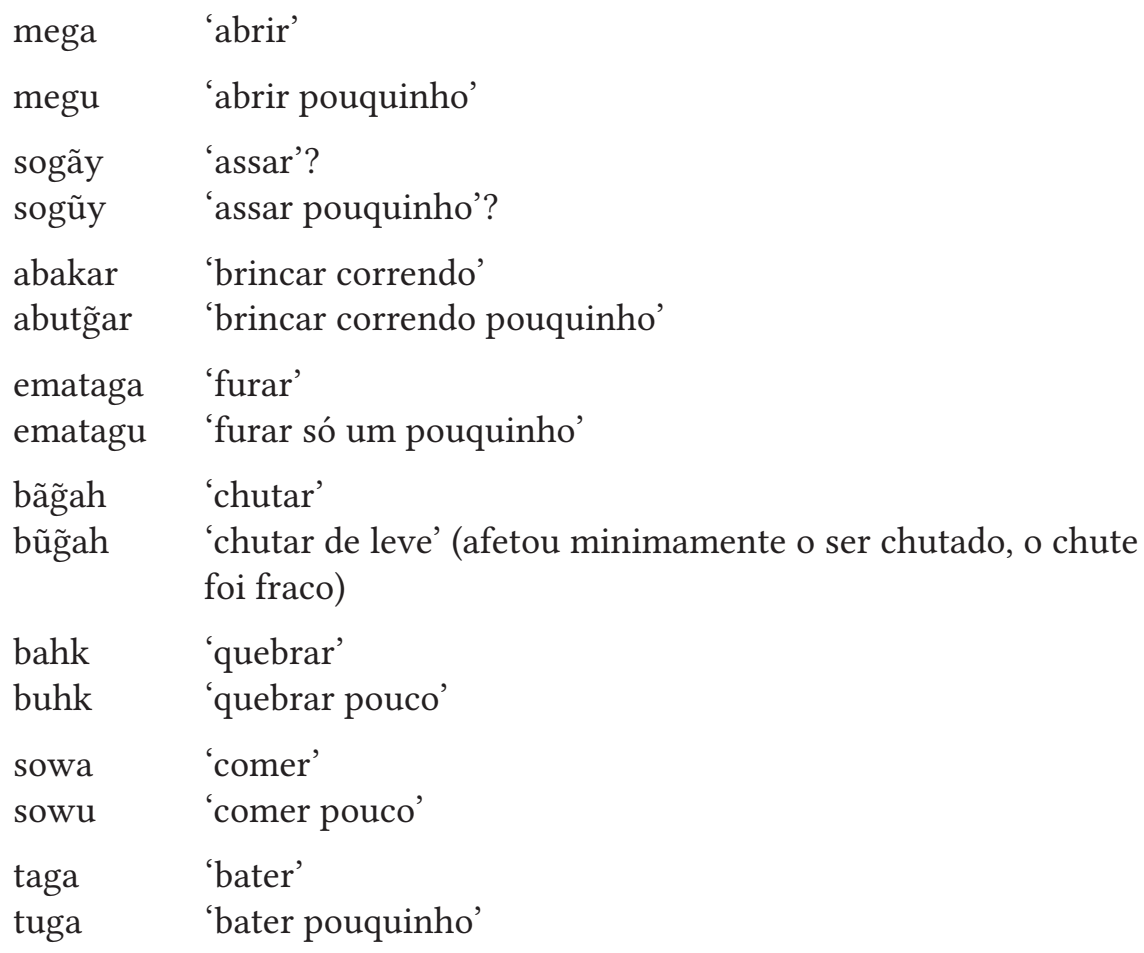

Nos exemplos que seguem, a atenuação envolve também a palatalização da consoante inicial do tema, se $d$, muda para $j$, se $t$, para $t x$, se $s$ para $x$, diante do vocalismo $(u)$ :

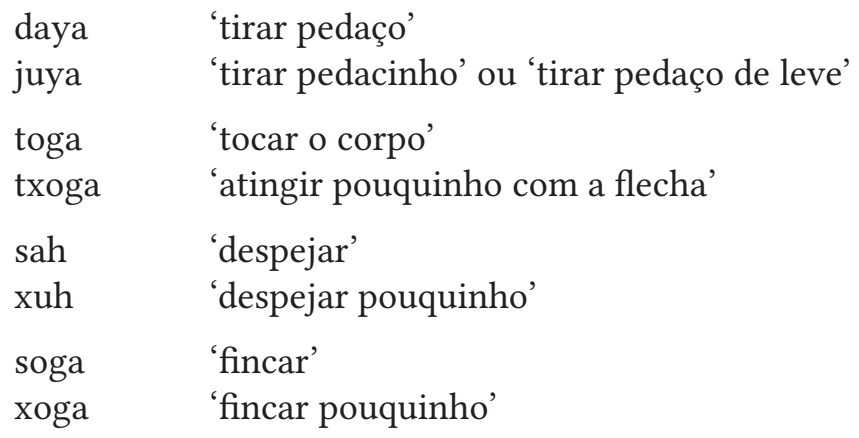


sõhrã 'enfiar'

xõhrã 'enfiar pouquinho'

É interessante notar que a maioria dos exemplos acima apresenta a palatalização diante da vogal $u$, o que indica que a vogal é o acionador da palatalização, mas, nos últimos três exemplos, a palatalização se dá diante da vogal $o$.

Note-se que há casos de atenuação de palavras trissilábicas em que o ablaut pode deslanchar concomitantemente assimilação da vogal precedente e palatalização da consoante $t$, possibilitando duas formas alternativas atenuadas:

$\begin{array}{ll}\text { takap } & \text { 'beliscar' } \\ \text { takup } & \text { 'beliscar de leve' } \\ \text { txukup } & \text { 'beliscar de leve' (outra forma de falar a mesma coisa) }\end{array}$

Em um dos exemplos selecionados a vogal alvo do ablaut é ẽ e o resultado é $\tilde{\imath}$ e não $\tilde{u}$.

$\begin{array}{ll}\text { asabatẽ } & \text { 'correr' } \\ \text { asabatĩ } & \text { 'correr(zinho)' }\end{array}$

Apresentamos, em seguida, alguns exemplos contextualizados da expressão do atenuativo por ablaut em Suruí. Os exemplos mostram que a atenuação em verbos não expressa necessariamente concordância com a dimensão física pequena do referente do argumento objeto, sujeito ou agente, mas pode expressar também afetividade, polidez, respeito, entre outros. No exemplo em (a), o verbo é atenuado como tentativa de minimizar o sentir dor do objeto afetado. No exemplo em (b), a atenuação expressa carinho e respeito ao avô. Em (c), o verbo chorar foi atenuado para ressaltar que o choro foi de raiva e não de dor. No exemplo (d), o verbo brigar foi atenuado para acentuar que o pai brigou por uma razão precisa e por carinho e preocupação para com o filho. Em (e), o verbo festejar foi atenuado para expressar a beleza, a singeleza do cantar das araras. Finalmente, em (f), o que é atenuado é a posposição 'com', como uma forma polida e carinhosa de pedir para alguém trazer algo.

a) Ate ewaga, anineh oje emitxagu e (emitxaga)

'não chore! eu só te dei uma furadinha' ou 'só te furei levemente'

b) Omamõ sade soe mũ og̃ay e (soe mã)

'meu avô está contando historinha para mim'

c) Atãr eka de awagu e (awaga)

'ele chorou(zinho) porque estava bravo' 
d) Olop de atxũr og̃ay e (atãr) 'meu pai brigou(zinho) comigo'

e) Kasarey sade arixũ e (arixã) 'as araras estão festejando(inho)'

f) Oma ur itxu eor mã (itxa) 'venha com(zinho) meu arco'

\section{Atenuação em Nhandéwa Txiripá e em Mbyá Tambeopé}

Em línguas Guaraní como o Mbyá Tambeopé e o Nhandéwa Txiripá o atenuativo -?i ocorre com nomes e com verbos. Em Nhandéwa Txiripá certos nomes são atenuados com tanta frequência, que se entende a combinação como já se encontrando em estágio avançado de lexicalização, como são os casos de palavras como 'casa' e 'caminho'. Os exemplos seguintes mostram essas palavras nas duas línguas.

1) Mbyá Tambeopé:

a) djaa txe ro py 'vamos para minha casa'

b) a ta tape rupi 'vou pelo caminho'

2) Nhandéwa Txiripá:

a) djaa txe ro'i py 'vamos para minha casinha'

b) aa ta tape'i rupi 'vou pelo caminhosinho'

É, assim, muito comum o uso do atenuativo em nomes como os seguintes no Nhandéwa Txiripá:

$\begin{array}{ll}\text { txigyre'i } & \text { 'tatuzinho' } \\ \text { worita'i } & \text { 'bolinhazinha' } \\ \text { retxutxa'i } & \text { 'corujinha' } \\ \text { maino'i } & \text { 'beija-florzinho' } \\ \text { kunhãtai'i } & \text { 'mocinha' } \\ \text { ywoty'i } & \text { 'florzinha' } \\ \text { waimi'i } & \text { 'velhinha' } \\ \text { tudja'i } & \text { 'velhinho' } \\ \text { kangue'i } & \text { 'ossadinha' } \\ \text { monde'i } & \text { 'armadilhazinha' } \\ \text { awatxi'ygue'i } & \text { 'espiguinha de milho' }\end{array}$




\section{Exemplos contextualizados com o atenuativo em Mbyá Tambeopé ${ }^{7}$}

Os exemplos seguintes foram extraídos de um relato mítico em NhandéwaTambeopé proferido por Wera Kwaray, residente na aldeia Tekoa Porã, conhecida em Português como Boa Esperança, no estado do Espírito Santo.

\section{Combinação do atenuativo com nomes}

mamo tu txetxy kãgue'i, e’i dje

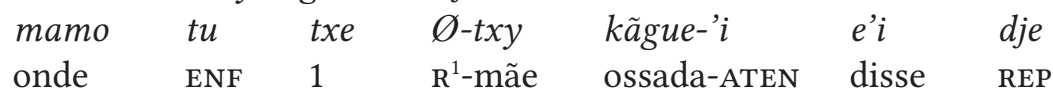

'onde está a ossada da minha mãe? Disse, contam'

a'ewy dje odjou a'e py ipuru'a'i wa'e iny

a'ewy dje odjou a'e py i-puru'a-'i wa'e iny então REP 3-encontrar esse LOC $\mathrm{R}^{2}$-grávida-ATEN NP PERM 'então, contam, encontrou a grávida que estava nesse local'

nhanderu oetxauka raka'e petei tape po'i'i

nhande $r$-u o-etxa-uka raka'e petei t-ape po'i-'i

1INCL. $\mathrm{R}^{1}$-pai 3-ver-PREP at.-PASS.REM um $\mathrm{R}^{4}$-caminho estreito-ATEN 'o nosso Deus mostrou um caminho estreitinho'

ah, ã̃ ma txe anho'i waiete ma aiko

ah a y ma txe anho-' $i$ waiete ma a-iko

INTERJ agora MD 1 soment-ATEN INTENS md 1-existir

'ah, agora estou muito sozinho'

\section{Combinação do atenuativo com verbo intransitivo}

tapytu'u'i ranhe txapy'a'i

t-apytu'u-i' ranhe txapy'a-'i

perm-descansar-ATEN primeiro instante-ATEN

'primeiro deixa eu descansar um instante'

\section{Combinação do atenuativo com verbos transitivos}

aỹ ma aa ta aikuaa pota'i tewe

a y ma a-a ta a-ikuaa pota-'i tewe agora MD 1-ir PROJ2 1-saber tratar-ATEN possibilidade 'agora eu vou tratar de saber, (o que existe ao redor)'

7 Abreviaturas usadas neste estudo: ATEN = atenuativo; ENF = enfático; GER = gerúndio; INCL. = inclusivo; INTERJ = interjeição; INTENS = intensivo; LOC = locativo; $\mathrm{MD}$ = marca discursiva; RED = reduplicação; REP = repetitivo; PASS.REM = passado remoto; PERM = permansivo; $\mathrm{PREP}=$ prepositivo PROJ = projetivo; $\mathrm{R}=$ prefixo relacional; $\mathrm{REP}=$ repetitivo . 
ywy omboai'i oikowy wa'erã ombodjera raka'e tatu worita'i

ywy o-mboai-'i o-iko-wy wa'e-rã o-mbo-djera raka'e

terra 3-corromper-ATEN 3-existir-GER NP-PROJ1 3-CAUS-criar AT-PASS.REM

tatu warita-'i

tatu bola-ATEN

'para corromper a terra (ia gerar), naquele tempo, (foi) tatu bolinhazinha'

\section{Combinação do atenuativo com partícula aspectual}

monde kora, mondeyke (dja) ope-ope rei'i ma

\begin{tabular}{|c|c|c|c|c|}
\hline & $\begin{array}{l}\text { kora } \\
\text { cercado }\end{array}$ & $\begin{array}{l}\text { monde-yke } \\
\text { armadilha-de.lado }\end{array}$ & $d j o$ & $\begin{array}{l}\text { o-pe-ope } \\
\text { 3-quebrar-RED }\end{array}$ \\
\hline
\end{tabular}

rei-'i ma

à.toa-ATEN MD

'o cercado da armadilha está de lado e meio quebrado'

\section{Combinação do atenuativo com palavra adverbial}

ã̃ ma djaa mombyry-mbyrywe'i, e'i

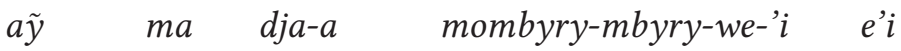

agora MD 12(3)-ir longe-RED-AD-ATEN disse

'agora vamos mais para longe, disse'

\section{Algumas considerações finais}

Neste estudo abordamos a expressão de atenuativo em três línguas de famílias distintas do tronco Tupí, Mbyá, Nhandéwa e Suruí, apontando para semelhanças na distribuição e funcionalidade dessa categoria nessas línguas. Colocamos em relevo o principal meio de expressão do atenuativo em Suruí, o processo de ablaut, por tratar-se de processo morfofonológico incomum no agrupamento genético Tupí. Consideramos esse processo como expressão de simbolismo sonoro, por sua produtividade. Hinton, Nichols e Ohala (1994:7) fazem as seguintes considerações sobre produtividade e simbolismo sonoro:

Certainly consonant or vowel substitution is one of the most common means for producing sound-symbolic expression, but only if a nonarbitrary (either natural or conventional) relationship between a segment and its meaning can be demonstrated would we want to call the process sound-symbolic. Nevertheless, a productive process of ablaut has the potential to be a process of conventional sound symbolism.

Indicações sincrônicas, como palatalização de coronais $t, d, n$ e $s$ diante de $u$ resultante do processo de ablaut sugerem que esse $u$ seja reflexo de um 
antigo $i$, e que, mesmo depois de sua mudança em $u$ o resultado fonológico da palatalização antiga dessas consoantes diante do antigo $i$ se manteve. Tendo sido esse o caso, há a possibilidade de o $u$ resultante do ablaut em Suruí ser cognato do morfemas -'i do Nhandéwa Txiripá e do Mbyá Tambeopé. Esse morfema $i$ do Suruí teria sobrevivido em exemplos como asabatẽ 'correr' : asabatĩ 'correr(zinho)'; aahta 'estar deitado' : aut i 'estar deitadinho'; ãnota 'estar em pé' : ãnyot ĩ 'estar em pe(zinho)'.

Mostramos também no presente estudo que nas três línguas o atenuativo não é exclusivo de nomes, mas tem como escopo diferentes classes de palavras. Também nessas línguas o atenuativo não se relaciona apenas com a dimensão física pequena, mas atenua tanto dimensões físicas quanto psicológicas, e pode atenuar a semântica de estados, eventos e processos. A motivação da atenuação é pragmática e seu uso controlado pelo falante.

\section{Referências}

Fortune, David Lee. 1985. Porto Velho Work Papers. Brasília: SIL.

Hinton, L.; Nichols, J.; Ohala, J. 1994. Sound Symbolism. New York: Cambridge University Press.

Jakobsen, William H. 1994. Nootkan vocative vocalism and its implications. In: Hinton, L.; Nichols, J.; Ohala, J. (eds.), Sound Symbolism, New York: Cambridge University Press pp. 23-39.

Jesperson, O. 1933. Symbolic value of the vowel i. In: Linguistica. Selected Papers in English, French and German. Copenhagen: Levin \& Munksgaard, p. 283-303.

Meer, Tine H. van der. 1985. Case marking in Surui. In: David Lee Fortune (ed.), Porto Velho Work Papers, p. 208-230. Brasília: SIL.

Sapir, E. 1911. Diminutive and augmentative consonant symbolism in Wishram. In: Handbook of American Indian Languages. Bureau of American Ethnography, Bull. 40, Part 1: 638-646, Washington, DC.

Sapir, E. 1929. Nootka baby words. International Journal of American Linguistics 5: 118-9.

Silverstein, M. 1994. Relative motivation in denotational and indexical sound symbolism of Wasco-Wishram Chinookan. In: Hinton, L.; Nichols, J.; Ohala, J. (eds.), Sound Symbolism, New York: Cambridge University Press, pp. 40-103.

Ultan, R. 1978. Size-sound symbolism. In Joseph Greenberg, (ed) Universals of Human Language, volume 2: Phonology. Stanford: University Press. 